\title{
Divination et prophétie : des pratiques oraculaires à leurs représentations dans la littérature française
}

\section{Arnaud Sibille}

\section{(2) OpenEdition}

1 Journals

Édition électronique

URL : http://journals.openedition.org/questes/3448

DOI : 10.4000/questes.3448

ISSN : 2109-9472

Éditeur

Les Amis de Questes

\section{Édition imprimée}

Date de publication : 15 juin 2014

Pagination : 53-72

ISSN : 2102-7188

\section{Référence électronique}

Arnaud Sibille, « Divination et prophétie: des pratiques oraculaires à leurs représentations dans la littérature française », Questes [En ligne], 28 | 2014, mis en ligne le 15 juin 2014, consulté le 10 décembre 2020. URL : http://journals.openedition.org/questes/3448 ; DOI : https://doi.org/10.4000/ questes.3448 


\title{
Divination et prophétie : des pratiques oraculaires à leurs représentations dans la littérature française
}

\author{
Arnaud SibILLE \\ Université Paris-Sorbonne (Paris IV)
}

La connaissance de l'avenir ou plutôt des vicissitudes qui ponctuent l'existence est, semble-t-il, un savoir particulièrement prisé et recherché, autour duquel se cristallisent les pires craintes, comme le soulignent les terreurs millénaristes qui caractérisent aussi bien le Moyen Âge que les temps modernes et qui sont portées par des hommes faisant souvent figure de prophète ou de devin. En l'an mil, la terreur eschatologique de la fin des temps, promise par les textes sacrés dans un délai que l'entendement humain n'est pourtant pas en mesure de déterminer, aurait animé simultanément l'esprit de foi et l'esprit de raison, ce qui se manifeste effectivement dans l'ambivalence de l'un des arts du quadrivium: l'astronomie, indissociable de sa sœur, l'astrologie. D'un côté, cette science permet de calculer les conjonctions planétaires, les éclipses ou encore d'établir les calendriers; de l'autre, il s'agit également d'un art divinatoire qui s'efforce de déchiffrer les mystères de l'avenir par l'observation des astres. Or, l'astronomie et l'astrologie sont bien toutes deux autant de réponses adressées à la peur de l'inconnu que suscitent les temps futurs, si bien qu'il est parfois difficile de les dissocier clairement ${ }^{1}$.

\footnotetext{
${ }^{1}$ C'est d'ailleurs ce à quoi s'attèle Nicole Oresme au XIV siècle dans le Livre de divinacions, en reconnaissant sans ambiguïté la noblesse de l'astronomie par opposition à l'errance astrologique : «Plusieurs ars ou sciences sont, par lesqueles on
} 
Deux conceptions de l'avenir se font donc concurrence : l'une, que l'on qualifiera de scientifique et qui émane de l'astronomie, déchiffrant dans l'univers des lois auxquelles sont soumis les corps naturels; l'autre, qui peut être considérée comme proche de la pensée magique et qui dérive de l'astrologie ainsi que des croyances eschatologiques, croyant voir dans le ciel les signes d'un destin inéluctable. Ces deux perspectives ne s'opposent pas pour autant, puisque l'esprit médiéval cherche à concilier sa soif de science avec une conception du monde héritée de la pensée chrétienne. C'est là, sans doute, l'une des premières raisons qui expliquent la coexistence des prophètes et des devins dans la littérature médiévale, les premiers se rapprochant plutôt de la pensée magique parce qu'ils croient tirer leurs oracles d'une inspiration, les seconds étant plus proches de la pensée rationnelle en ce qu'ils font usage de techniques et de savoirs ${ }^{2}$. Puisqu'un nombre relativement important de manuscrits divinatoires a été conservé, il est possible de comparer la pratique divinatoire telle qu'elle est à l'œuvre dans les livres des devins et les techniques oraculaires telles

seult enquerir des choses avenir, ou occultes, secretes, mucies, ou qui a ce peuent estre appliquees. L'une est astrologie, laquelle, ce me samble, a aussi comme six principales parties. La premiere determine principalement des mouvemens, des signes, et des mesures des corps du ciel, par laquelle avec les tables on puet savoir les constellacions et les eclipses avenir et samblables choses. [...] La quinte est de interrogacions a jugier et respondre d'une question par la constellacion qui est ou ciel en l'eure de la demande. La sixte est de elections pour eslire heure de commencier un voyage ou une besongne, et soubz ceste partie est contenue celle qui enseigne a faire ymages, carettes, aneaux, et telx choses. [...] La premiere partie d'astrologie est speculative et mathematique, tres noble et tres excellente science, et baillie es livres moult soubtilment et la puet on suffisament savoir, mais ce ne puet estre precisement et a point, si comme j'ay declaire en mon traictie de la Mesure des Mouvemens du Ciel et l'ay prouve par raison fondee sur demoustracion mathematique. [...] La quinte partie, des interrogacions, et la sixte, des elections, n'ont point de raisonnable fondement et n'y a point de verite. » George Coopland, Nicole Oresme and the astrologers: A study of his Livre de divinacions, Cambridge (Mass.), Harvard University Press, 1952, p. 52.

${ }^{2}$ Cette distinction entre divination inspirée, issue des songes et des prophéties, et divination déductive, qui résulte de l'interprétation de signes selon une technique particulière, est devenue classique grâce à Jean Bottéro, Mésopotamie. L'écriture, la raison et les dieux, Paris, Gallimard, 1987. 
qu'elles sont représentées dans la littérature de l'époque: les ouvrages littéraires restituent-ils une image fidèle et complète de la divination médiévale ? En outre, la question des rapports entre divination et prophétie se pose puisque toutes deux fonctionnent comme des ressorts narratifs, en particulier en ce qui concerne les chansons de geste : quel bénéfice peut-on tirer de la coexistence ou de la concurrence entre divination et prophétie ?

\section{La connaissance de l'avenir : un savoir magique?}

Les devins et les prophètes ne sont pas les seuls en mesure de prédire l'avenir, dans cette culture médiévale caractérisée en partie par le goût de la merveille. Les enchanteurs, en effet, s'illustrent également par une connaissance intuitive des événements, qui ne provient donc ni d'un calcul sur la conjonction des astres, ni d'une inspiration communiquée par la Providence : ni devins, ni prophètes, ces enchanteurs ont recours à la magie et leur fait est bien connu des hommes. C'est pourquoi Yseut songe à l'intervention d'un magicien dans la découverte de son plus intime secret, dans la Folie Tristan d'Oxford, lorsque le « fou » récemment arrivé à la cour du roi Marc révèle ce qui n'était connu que de la reine, de son amant et de sa confidente ${ }^{3}$. Si Yseut est victime de sa propre ignorance parce qu'elle n'a pas reconnu Tristan ainsi grimé, elle élabore néanmoins, sous l'effet de l'étonnement, une hypothèse plausible : la connaissance du secret qu'a montrée le curieux personnage à la cour du roi ne peut venir que d'un adepte des sciences occultes. Comme l'indique l'emploi du terme enchantements, il ne s'agit pas ici d'une simple technique de divination mais de magie, laquelle peut elle aussi s'efforcer de découvrir les mystères

\footnotetext{
${ }^{3}$ « Certes, Brengain, mult me merveil /Ki li descuvri mun cunseil. /Kar nus nel sout fors je e vus /E Tristran, le cunseil de nus. /Mais cist tafur, men escient, /Le set tut par enchantement. » Folie Tristan d'Oxford, éd. Ernest Hoepffner, Paris, Belles Lettres coll. «Publications de la Faculté des lettres de l'Université de Strasbourg. Textes d'étude », 8, 2 éd., 1943, v. 567-572.
} 
du passé et de l'avenir ${ }^{4}$. Alors que le mode opératoire de la magie implique l'invocation et la manipulation d'esprits pour agir sur le monde ${ }^{5}$, la divination en revanche, telle que la définit Auguste Bouché-Leclerq dans son Histoire de la divination dans l'Antiquité, « a pour domaine tout ce que l'esprit humain ne peut connaître par ses seules forces, en premier lieu l'avenir, en tant qu'il échappe à la prévision rationnelle ». Si dans les deux cas il est bien question d'une opération surnaturelle, il convient pourtant de définir avec netteté la frontière entre divination et magie, afin de comprendre pourquoi nous ne parlerons pas des enchanteurs et des magiciens, mais seulement des devins et des prophètes.

Le terme enchantements a une extension plus large en effet que celui de divinations, ce qui s'explique d'abord par les possibilités virtuellement illimitées qu'offre la magie, tandis que les arts divinatoires se limitent à la connaissance d'événements passés ou futurs par un ensemble de techniques auxquelles peut se livrer l'entendement humain, sans la médiation d'esprits. Cette différence de nature entre arts magiques et divinatoires n'est pas négligeable car elle permet de rendre compte de la relative tolérance de l'Église face à la divination ${ }^{6}$ : la connaissance de l'avenir est surtout considérée comme un péché de vanité, alors que le dogme condamne fermement la nigromance qui fait appel aux démons. La

\footnotetext{
${ }^{4}$ L'étude d'un ouvrage de nigromance montre par exemple qu'un nombre important de rituels magiques ont un rôle divinatoire. Dans le manuscrit de nigromance édité par Richard Kieckhefer (Forbidden rites : a Necromancer's manual of the fifteenth century, Stroud, Sutton, 1997), dix-neuf rituels sur plus d'une centaine se proposent de révéler l'identité d'un voleur ou l'endroit où se cache un butin, questions ordinaires posées aux arts divinatoires.

${ }^{5}$ Pour plus de précisions sur les questions ayant trait à la magie médiévale, on se reportera avec profit à Richard Kieckhefer, Magic in the Middle Ages, Cambridge, Cambridge University Press, 1993 ; Claire Fanger, Conjuring spirits : texts and traditions of late medieval ritual magic, Stroud, Sutton, 1999 ; Jean-Patrice Boudet, Entre science et nigromance. Astrologie, divination et magie dans l'Occident médiéval (XII $-X V^{e}$ siècles), Paris, Publications de la Sorbonne, 2006.

${ }^{6}$ Sur cette question, voir en particulier Pierre Boglioni, «L'Église et la divination au Moyen Âge, ou les avatars d'une pastorale ambiguë », Théologiques, 8, $\mathrm{n}^{\mathrm{o}} 1,2000$, p. 37-66.
} 
frontière entre divination et magie néanmoins n'est pas toujours aussi nette dans la littérature que dans la législation ecclésiastique ${ }^{7}$, comme le souligne en particulier l'ambiguïté du sens donné dans les textes littéraires au terme de nigromance : d'une part, il peut faire référence à la magie rituelle ayant cours au Moyen $\hat{A g e}^{8}$, qui procède par invocation d'esprits et qui se réclamerait alors de l'étymon niger manteia ${ }^{9}$, « magie noire »; d'autre part, la nigromance peut désigner la technique divinatoire qui en appelle à l'esprit des morts pour obtenir des réponses aux questions des vivants, se réclamant alors de l'étymon $v \varepsilon \kappa \rho о \mu \alpha \nu \tau \varepsilon i ́ \alpha$. Or, ce terme de nigromance habite les textes littéraires médiévaux jusqu'à qualifier - de manière exceptionnelle il est vrai-1'art utilisé par un devin. Aussi le savoir astronomique de l'enchanteur Malaquin lui permet-il de déverrouiller la porte d'un donjon ${ }^{10}$ : nous sommes bien loin de l'astrologie généthliaque ou de l'astronomie tournée vers l'observation des corps célestes et la littérature ne semble guère faire de différence entre astrologiens et nigromanciens.

Dans les faits en revanche, le savoir magique en général et « nigromantique » en particulier est d'une nature absolument distincte de la divination ou de la prophétie: la nigromance en appelle à des forces

\footnotetext{
${ }^{7}$ Richard Kieckhefer, Magic in the Middle Ages, op. cit., p. 12.

${ }^{8}$ On prendra soin néanmoins de bien distinguer nigromance et ars notoria. Toutes deux constituent bien des pratiques magiques, mais alors que la première en appelle aux démons, la seconde sollicite la bienveillance des anges. De plus, le but recherché par les rituels diffère considérablement d'une technique à l'autre. Voir à ce propos Julien Véronèse, L'Ars notoria au Moyen Âge. Introduction et édition critique, Florence, Sismel - Edizioni del Galluzzo, 2007 ; Katy Sarah Bernard, «Compter, dire et figurer: édition et commentaire de textes divinatoires et magiques en occitan médiéval », thèse de doctorat, Université Michel de Montaigne-Bordeaux III, 2008, p. 107-108.

${ }^{9}$ Jean-Patrice Boudet, Entre science et nigromance. Astrologie, divination et magie dans l'Occident médiéval (XII $-X V^{e}$ siècles), op. cit., p. 92-94.

10 « La trueve un posterne des le tans establie./Li lerres la defferme par l'art d'astronomie ", Jehan de Lanson, chanson de geste of the 13th Century, edited after the manuscripts of Paris and Bern with introduction, notes, table of proper names and glossary, éd. J. V. Myers, Chapel Hill, The University of North Carolina Press, 1965 , v. 2391-2392.
} 
surnaturelles et est à l'initiative d'un individu, tandis que le prophète est le réceptacle de l'inspiration et le devin, s'il est bien à la source de la prédiction, ne s'appuie sur rien d'autre que sur ses observations et ses calculs. Cette différence ontologique explique pourquoi si peu d'ouvrages «nigromantiques» ont échappé aux flammes, alors que l'on retrouve encore un nombre important de manuscrits divinatoires. Dans ces conditions, seuls les textes ayant appartenu à des devins et non à des nigromanciens retiendront notre attention, puisqu'il est possible de comparer les textes pratiqués par les devins avec leurs représentations littéraires médiévales.

\section{De la divination à la littérature}

Si la littérature peut, à la manière d'un miroir, refléter les arts divinatoires en pratique au cours du Moyen Âge, ce miroir peut aussi être déformant ou bien n'offrir qu'un reflet partiel. Aussi est-il important de rappeler d'abord la diversité des techniques oraculaires qui ont cours en Occident avant d'examiner la fidélité de leurs représentations littéraires.

Jusqu'au XII ${ }^{\mathrm{e}}$ siècle, la divination proprement occidentale s'appuie sur l'astrologie, l'arithmancie, l'oniromancie, la cléromancie et les pronostics. L'astrologie pratiquée jusqu'alors est assez rudimentaire : ses opuscules portent le nom de lunaria et zodiologia, textes brefs qui établissent des prédictions grâce à l'observation de la lune ou de la position de la lune dans le zodiaque ${ }^{11}$ et qui se réclament souvent d'une figure tutélaire biblique, comme Daniel ou encore Salomon. L'arithmancie est aussi une technique fort en vogue, comme le prouve le succès de la «sphère de Pythagore », aussi appelée «sphère d'Apulée » ou encore « sphère de Pétosiris », que l'on retrouve dans une quantité importante de

${ }^{11}$ Pour une étude approfondie de ce corpus, voir Emanuel Svenberg, Lunaria et Zodiologia Latina, Göteborg, Acta Universitatis Gotoburgensis, 1963. 
manuscrits $^{12}$ : elle consiste à traduire en chiffres les lettres du prénom et du nom de la personne qui consulte, ainsi que celles du nom et du prénom de la mère du consultant, ce qui produit une somme à diviser à plusieurs reprises. Le chiffre restant se situe alors dans la fameuse « sphère », divisée en son milieu par une ligne horizontale: si le chiffre se retrouve dans l'hémisphère supérieur, la réponse est favorable et présage la survie, mais s'il est situé dans la partie inférieure, la réponse est défavorable et prédit la mort. L'oniromancie jouit également d'une certaine popularité, en particulier grâce aux Somnia Danielis, clef des songes permettant de comprendre la dimension prémonitoire de ceux-ci ${ }^{13}$. Dans ce cas, la matière $\mathrm{du}$ texte revêt une coloration plus religieuse que celle des autres textes divinatoires contemporains, puisqu'elle s'inscrit dans la tradition des songes envoyés par Dieu au prophète Daniel. La cléromancie est une autre pratique divinatoire largement utilisée en Occident et qui elle aussi a été très tôt contaminée par la religion : dans sa forme la plus rudimentaire en effet, il s'agissait de lire au hasard une phrase de la Bible ${ }^{14}$, à laquelle on accordait une valeur oraculaire ${ }^{15}$. Par la suite, des ouvrages divinatoires spécifiques voient le jour, notamment les Sortes apostolorum ${ }^{16}$, les « sorts des Apôtres », aux côtés de livres de sorts profanes. Enfin, une autre

${ }^{12}$ Henry Sigerist, «The "Sphere of Life and Death" in Early Mediaeval Manuscripts », Bulletin of the History of Medicine, 11, 1942, p. 292-303 ; Linda Ehrsam Voigts, "The Latin verse and Middle English Prose Texts on the Sphere of Life and Death in Harley $3719 »$, The Chaucer Review, 21, n 2, 1986, p. 291-305.

${ }^{13}$ Lorenzo Di Tommaso, The Book of Daniel and the Apocryphal Daniel Literature, Leiden - Boston, Brill, 2005, p. 231-259 et p. 378-402.

${ }^{14}$ C'est ce que l'on appelle sorts bibliques ou encore consultations des Évangiles. Voir en particulier Pierre Boglioni, art. cit., p. 51-55.

${ }^{15}$ Pour plus de précisions concernant l'histoire du genre des livres de sorts, voir Solange Lemaître-Provost, Les livres de sorts en Moyen Français. Étude et édition critique, thèse de doctorat, Département des Littératures, Faculté des Lettres, Université Laval, Québec, 2010.

${ }^{16}$ Pour un état de la question sur les livres de sorts chrétiens en général et les Sortes apostolorum en particulier, voir William E. Klingshirn, «Defining the Sortes Sanctorum: Gibbon, du Cange, and Early Christian Lot Divination », Journal of Early Christian Studies, 10-1, 2002, p. 77-130. 
tradition ancienne tire son origine du prophète Esdras auquel Dieu aurait enseigné la lecture des signes : il s'agit des pronostics, qui dévoilent les grandes lignes de l'année en fonction du jour sur lequel tombe le premier janvier $^{17}$. Parfois, ce ne sont pas les calendes de janvier qui sont prises comme jour de référence, mais Noël, et il n'est pas toujours question d'Esdras mais aussi d'Ezéchiel. Un autre type de pronostics prête aussi tout particulièrement attention au tonnerre ${ }^{18}$.

Avec l'arrivée en Europe des textes arabes à partir du XII ${ }^{\mathrm{e}}$ siècle, les pratiques oraculaires se diversifient et se complexifient. Ainsi, l'astrologie est considérablement renouvelée et les opuscules astrologiques revendiquent ostensiblement dans leur titre une origine arabe, ce peuple étant réputé maître en la matière ${ }^{19}$. Ce renouveau de l'astrologie est en particulier provoqué par l'arrivée en Europe du Tetrabiblos de Ptolémée, traduit d'abord du grec en arabe sous le nom de Kitāb al-arba 'a, puis de l'arabe en latin sous le titre de Quadripartitum ${ }^{20}$. L'astrologie s'intègre alors dans la pratique de la médecine, en déterminant par exemple les jours fastes et néfastes pendant lesquels il faut saigner le patient ou au contraire le laisser

${ }^{17} \mathrm{Cf}$. François Nau, « Analyse de deux opuscules astrologiques attribués au prophète Esdras et d'un calendrier lunaire de l'Ancien Testament attribué à Esdras, aux Egyptiens et même à Aristote », Revue de l'Orient chrétien, II, 12, 1907, p. 14-21; Françoise Féry-Hue, "Revelatio Esdrae ou Prophéties d'Ezéchiel. Eléments nouveaux pour le corpus latin et français des prophéties d'après le jour de Noël », dans "Pour acquérir honneur et pris». Mélanges de Moyen Français offerts à Giuseppe Di Stefano, dir. Maria Colombo Tinelli et Claudio Galderisi, Montréal, Ceres, 2004, p. 237-251.

18 Jean-Patrice Boudet, Lire dans le ciel. La Bibliothèque de Simon de Phares, astrologue du XV $V^{e}$ siècle, Bruxelles, Centre d'étude des manuscrits, 1994, p. 121.

${ }^{19}$ Pour n'en citer que quelques-uns, nous trouvons : Li exemple que Hally met en plusieurs nativités (Paris, BnF, fr. $1349, \mathrm{f}^{0} 213 \mathrm{r}^{\circ} \mathrm{a}-214 \mathrm{v}^{\circ} \mathrm{b}, \mathrm{XIV}^{\mathrm{e}}$ siècle), Le traité Messehallac pour savoir la pensee et la cogitacion d'aucun (Cambridge (Mass.), Harvard University Library, Houghton, French 151, f $\mathrm{f}^{\mathrm{6}} 68-69, \mathrm{XV}^{\mathrm{e}}$ siècle), L'Espitre Messalla sur la nature, significacion des XII signes et VII planetes et eclipses (Paris, $\mathrm{BnF}$, Bibliothèque de l'Arsenal, 2872, $\mathrm{f}^{0} 79 \mathrm{v}^{\mathrm{o}} \mathrm{a}-83 \mathrm{r}^{\circ} \mathrm{a}, \mathrm{XIV}^{\mathrm{e}}$ siècle), etc.

${ }^{20}$ Sur ces questions, voir Olaf Pedersen, A Survey of the Almagest, Odense, Odense University Press, 1974. 
en convalescence ${ }^{21}$. De nouvelles techniques apparaissent aussi, telles que la chiromancie ou la géomancie. Si la première est bien connue, la seconde en revanche l'est beaucoup moins, alors même qu'elle fut utilisée de manière extensive dans tout l'Occident médiéval: en s'appuyant sur un fondement astrologique, cette technique consiste simplement à tracer de manière aléatoire des lignes de points qui, après avoir été simplifiées, produisent quatre symboles. À partir de ces quatre signes, il est possible d'établir un thème constitué de quinze figures géomantiques qui permettent de répondre aux questions du consultant ${ }^{22}$. Enfin, la physiognomonie fait également son entrée dans l'Occident médiéval, entendant prédire l'avenir d'un individu et sa future apparence en fonction de sa planète d'élection lors du jour de sa naissance.

Malgré la grande diversité des arts divinatoires, la littérature médiévale procède à une simplification à l'extrême de cet ensemble de pratiques. Ainsi, il est excessivement rare que nous ayons des précisions sur la technique divinatoire employée, comme si cela n'avait guère d'importance aux yeux du narrateur. L'expression la plus souvent utilisée lorsque le devin se livre à sa besogne est « jeter les $\operatorname{sorts}^{23}$ », les « sorts » ayant ici le sens de «tirage au sort» comme dans les Sortes apostolorum dont nous avons déjà parlé, et non pas le sens actuel de «sortilège ». Il arrive néanmoins qu'en de rares occasions l'auteur décrive avec précision

${ }^{21}$ De nombreux ouvrages sont déjà consacrés à cette question. Outre les incontournables de Danielle Jacquart, on pourra consulter avec profit Pearl Kibre, " "Astronomia" or "Astrologia Ypocratis" ", dans Science and History : Studies in Honor of Edward Rosen, dir. P. Czartoryski, E. Hilfstein et F. D. Grande, Varsovie, The Polish Academy of Science Press, 1978, p. 133-156, Pearl Kibre s'intéressant ici au Libellus de medicorum astrologia attribué au Pseudo-Hippocrate.

${ }^{22}$ Le détail de la méthode géomantique est exposé dans une thèse qui a fait date: Thérèse Charmasson, Recherches sur une technique divinatoire : la géomancie dans l'Occident médiéval, Genève, Droz, 1980.

${ }^{23}$ En ce qui concerne la littérature médiévale en langue française, c'est la conclusion à laquelle aboutit Solange Lemaitre-Provost, op. cit., p. 159-171. En revanche, il semblerait que la littérature occitane donne plus de détails, voir Katy Sarah Bernard, op. cit., p. 663-674. 
la scène de divination, comme cela est le cas dans le Moniage Guillaume $^{24}$ : les clercs de Synagon en effet «getent lor sort parmi une fenestre ${ }^{25} »$, c'est-à-dire qu'ils jettent dans ce cas présent quelques brins de paille. Ensuite, grâce à la disposition des différents brins sur le sol et à leur direction, les clercs sont en mesure de prédire à Synagon sa défaite prochaine contre les Français. L'originalité de cet exemple ne réside pas seulement en ce qu'il décrit avec précision la méthode divinatoire et l'interprétation qui en est tirée, mais encore en ce que cette pratique oraculaire n'est pas attestée dans les textes divinatoires de l'époque. Peutêtre s'agit-il d'une pratique rudimentaire de la géomancie, la position au sol des objets lancés revêtant alors une valeur oraculaire, ce qui s'apparenterait à des techniques encore en pratique, en Afrique notamment. Peut-être estce aussi une création littéraire qui ne tire pas ses sources de l'observation du réel. Lorsque le devin ne jette pas seulement les sorts et que la narration le qualifie d'astrologue, les auteurs précisent de temps en temps qu'il se livre à l'observation des étoiles. En fait, la représentation de la divination qu'offre la littérature médiévale semble être pratiquement réduite à la pratique de l'astrologie et des sorts, puisqu'on ne rencontre qu'en de très rares occasions d'autres techniques oraculaires. En l'occurrence, la Chanson d'Aspremont présente une scène de catoptromancie, c'est-à-dire

${ }^{24}$ Nous empruntons cet exemple aux pages 405 et 406 de l'excellent article de JeanClaude Valecalle, "Remarques sur l'astrologie et la divination dans les chansons de geste », dans Le soleil, la lune et les étoiles au Moyen Âge, CUERMA, Aix-enProvence, Presses de 1'Université de Provence, 1983, p. 403-418.

${ }^{25}$ « La nuis est coie et la lune luist bele, /Getent lor sort parmi une fenestre, /Un riche paile li maistre clers i gete. /Desront li pailes et descire et deserre, /En quatre pars desront et escantele;/Les trois parties en cäirent a tere, /La quarte part se tient comme arondele, /En air se tient, ni ciet ne cancele. /Li clerc le voient, a poi d'ire ne dervent, /Cascuns tenoit sa main a sa maissele,/Pleurent et crient et font dure moleste. /Dist Synagons : "Que avés signor mestre ?/Que dist li sors ? Dites le moi, caele !" /"Sire," font il, "grant dolor et grant perte !" " Le Moniage Guillaume II, éd. W. Cloetta, t. I, Paris, Société des anciens textes français, 1906, v. 2911-2924. 
de divination par le miroir ${ }^{26}$ : le devin Balan révèle dans le reflet d'une glace le mouvement des armées et flottes sarrasines. Compte tenu de la rareté de cet art divinatoire, il se pourrait que la scène de catoptromancie de la Chanson d'Aspremont soit plutôt un écho littéraire au «miroir de Virgile $^{27} \gg$ que la description d'une technique couramment pratiquée dans la société médiévale. En revanche, alors même que la géomancie et la chiromancie jouissent d'une extrême popularité au Moyen Âge et qu'elles sont recopiées à de nombreuses reprises dans une grande quantité de manuscrits, il n'en est fait nullement mention dans les chansons de geste, jusqu'aux plus tardives. Il semble donc qu'il y ait un écart non négligeable entre les techniques divinatoires pratiquées dans la société médiévale et celles qui sont représentées dans le champ littéraire.

Cependant, la divination étant couramment pratiquée dans la société médiévale, la création littéraire s'en retrouve nécessairement affectée: certains motifs littéraires en effet, sans qu'ils ne revendiquent d'appartenance à une tradition divinatoire en particulier, rappellent sur le mode de la connivence les pratiques oraculaires utilisées à l'époque. Par

${ }^{26}$ Cette technique oraculaire est d'ailleurs très rare au Moyen Âge, l'un des seuls exemples, et parmi les plus célèbres, se trouve dans le Policraticus de Jean de Salisbury. Voir Jean-Patrice Boudet, Entre science et nigromance. Astrologie, divination et magie dans l'Occident médiéval (XII ${ }^{e}-X V^{e}$ siècles), op. cit., p. 69-71.

${ }^{27}$ Virgile à l'époque médiévale est moins connu comme poète que comme magicien ou savant. Jean de Salisbury est d'ailleurs le premier, dans le Policraticus, à attribuer au Virgile industrieux du Moyen Âge un prodige tout à fait exceptionnel : celui de la mouche d'airain. Naples, affligée par une invasion de mouches tueuses, est sauvée par la création de Virgile qui met un terme à la menace. Parmi les nombreuses inventions qui lui sont attribuées, comme la tête qui parle, le pont magique permettant d'aller où l'on veut, le château tournant, etc., le miroir qu'il fabrique est un charme particulièrement précieux qui s'apparente à la divination, en ce qu'il dévoile ce qui se trame dans le secret: "On lit dans le Livre des Sept Sages que Virgile fit placer sur une colonne un miroir très beau qui avait la propriété et l'avantage de révéler tout préparatif d'un royaume contre cette ville, ce qui permettait à ses citoyens de se protéger et de se préparer à la guerre; de telle sorte que grâce à ce miroir ils échappaient toujours à leurs ennemis ", Jean Gobi Le Jeune, Exemplum $n^{\circ} 817$, dans Marie Anne Polo De Beaulieu, Education, prédication et cultures au Moyen-Âge: Essais sur Jean Gobi le Jeune, Presses Universitaires de Lyon, 1999, p. 27. 
exemple, nous pourrions voir dans la Fontaine amoureuse de Guillaume de Machaut une réminiscence de la catoptromancie, qui, si elle était rare, n'en était pas moins pratiquée. Le poète en effet présente cette fontaine comme une source enchantée : boire de son eau, nous dit-il, suscite immédiatement l'amour ${ }^{28}$. Cependant, la description de la fontaine lui confère aussi une valeur prémonitoire : on peut voir dans le reflet de l'eau deux sculptures, l'une représentant l'histoire de Narcisse, l'autre la guerre de Troie en deux tableaux, l'un montrant Vénus qui enflamme Hélène, l'autre Achille qui affronte Hector. Au moment de se pencher pour boire à cette fontaine, l'amoureux en devenir est donc averti du sort qui l'attend et qu'il entrevoit dans le reflet de l'eau, laquelle se fait le miroir des scènes qui décorent la fontaine, comme si elles étaient toutes autant de visions de l'avenir. Un autre exemple de catoptromancie (mais il s'agit ici, plus spécifiquement, d'onychomancie, c'est-à-dire de divination par le reflet des ongles) se trouve dans le Jeu de la Feuillée, le médecin répondant, par l'intermédiaire de son apprenti, à la question de Dame Douche qui vient le consulter ${ }^{29}$. Le contexte satirique dans lequel s'insère cette mention de l'onychomancie montre aussi que l'on peut se moquer de la divination et des charlatans. Enfin, pour prendre un dernier exemple, un événement exceptionnel dans la Chanson de Roland peut rappeler le souvenir de la pratique divinatoire nommée brontoscopie, c'est-à-dire la divination par la foudre ${ }^{30}$. En effet, à la mort de Roland, une formidable tempête se déchaîne en France, cette vision apocalyptique annonçant en fait aux Français la calamité de la guerre

${ }^{28}$ Guillaume De Machaut, La Fontaine amoureuse, éd. Jacqueline Cerquiglini-Toulet, Paris, Stock, 1993, v. 1413.

29 «Li Fisisciens: Et j'en ferai warder ou pauc, /Pour acomplir vostre menchoigne. /Rainelet, il couvient c'on oigne/Ten pauc; lieve sus un petit. /Mais avant estuet c'on le nit. /Fait est. Rewarde en cheste crois /Et si di chou que tu i vois », Adam Le Bossu, Le Jeu de la Feuillée, éd. Ernest Langlois, Paris, Champion, 1984, v. 258-164.

${ }^{30}$ Pour plus de détails sur cette pratique divinatoire issue de l'antiquité, voir Stefan Weinstock, "Libri fulgurales », dans Papers of the British School at Rome, vol. 19, 1951, p. 122-153. 
en Espagne, avec la perte des pairs de France et de Roland; mais les Francs, faute de devins, interprètent mal le signe, voyant dans cet orage l'annonce de la fin des temps, alors qu'il ne s'agit que d'une défaite militaire, aussi lourde soit-elle ${ }^{31}$. La foudre est naturellement perçue comme une intervention divine et c'est sans doute la raison pour laquelle les premiers devins en ont fait un signe permettant de dévoiler l'avenir. S'il est impossible de savoir si la foudre dans la Chanson de Roland n'est qu'un présage divin ou est aussi un rappel des pratiques brontoscopiques que l'on retrouve en particulier dans les pronostics d'Esdras, il apparaît dans tous les cas que la création littéraire se nourrit aussi bien des images spectaculaires que des techniques divinatoires pratiquées durant le Moyen Âge.

\section{De la littérature à la prophétie}

Si la littérature médiévale ne donne pas toujours une représentation complète et précise des différentes techniques divinatoires, non seulement parce que cela, sans doute, n'est pas essentiel à l'œuvre mais également parce que le public connaît déjà celles-ci dans la mesure où les devins font partie de leur vie quotidienne, elle offre en revanche une vision représentative des différents types de personnages capables de prédire l'avenir: outre les nigromants et les devins dont nous avons déjà parlé, figurent les prophètes. Comparer divination et prophétie dans les textes littéraires revient donc à examiner l'opposition entre profane et religieux,

${ }^{31}$ « Franceis i perdent lor meillors guarnemenz, /Ne reverrunt lor pers ne lor parenz $/ \mathrm{Ne}$ Carlemagne ki as porz les atent. /En France en ad mult merveillus turment, /Orez i ad de tuneire e de vent, /Pluies e gresilz desmesureement, /Chiedent i fuildres e menut e suvent, /E terremoete ço $\mathrm{i}$ ad veirement./De Seint Michel del Peril josqu'as Seinz, /Des Besençun tresqu'as [porz] de Guitsand/Nen ad recét dunt li mur ne cravent. /Cuntre midi tenebres i ad granz, /N'i ad clartét, si li ciels nen i fent ; /Hume nel veit ki mult ne s'espaent. /Dient plusort : "Co est li definement, /La fin del secle ki nus est en present." /Il nel sevent, ne dient veir nient : /Co est li granz dulors por la mort de Rollant », La Chanson de Roland, éd. Pierre Jonin, Paris, Gallimard, 1979, v. $1420-1437$. 
puisque la prophétie, contrairement à la divination, suppose une élection divine, un «choix providentiel ${ }^{32} »$. Dans un système de pensée judéochrétien, la prophétie est par nature légitime, envoyée par Dieu, alors que la divination est nécessairement sujette à caution.

La diversité des types de personnage en mesure de prédire l'avenir tient à ce que la nature de la divination est double $^{33}$ : d'un côté, la divination est déductive, c'est-à-dire qu'elle est composée d'un ensemble de techniques par lesquelles l'être humain cherche à percer les secrets de l'avenir; de l'autre, la divination est inspirée dans le cas où l'homme bénéficie d'une révélation spontanée communiquée par la puissance divine. Cette distinction classique permet d'opposer la divination, qui est une technique et un savoir, à la prophétie, laquelle est le signe d'une élection providentielle ; pourtant, toutes deux sont des moyens d'accéder à ce que cache le futur. D'un côté, la démarche est prospective, l'homme cherchant à deviner par ses propres moyens ce que l'avenir lui réserve ; de l'autre, la prophétie survient sans avoir été recherchée. Dans les chansons de geste, cette différence de nature entre divination déductive et divination inspirée permet justement de construire l'opposition entre les belligérants, entre les Chrétiens et les Sarrasins. Ainsi, dans la Chanson de Roland, Charlemagne bénéficie de nombreux rêves prémonitoires, dont la signification n'est pas immédiatement claire, mais qui sont pourtant la préfiguration des événements à venir ${ }^{34}$. Le rêve est dans ce cas la marque distinctive du héros choisi par Dieu: il légitime le combat porté à l'encontre des Sarrasins. Ceux-ci en effet ne reçoivent aucun songe de Mahomet, d'Apollon ou de

${ }^{32}$ Jean-Claude Valecalle, op. cit., p. 414.

${ }^{33}$ Nous reprenons ici la terminologie et les catégories de l'ouvrage fondamental en la matière de Jean Bottéro, op. cit.

${ }^{34}$ On peut penser en particulier au songe de Charlemagne dans la laisse LVII, où l'empereur voit un ours, un léopard et un lévrier s'affronter dans la chapelle d'Aix, ce qui est la préfiguration du duel judiciaire entre Thierry et Pinabel qui permet à la fois de châtier Ganelon et de venger Roland: La Chanson de Roland, éd. cit., v. 725-736. 
Tervagant, fameuse trinité idolâtre attribuée aux Sarrasins. En revanche, ils ont dans leurs rangs des personnages dont ne disposent pas les Chrétiens : les devins. Et force est de reconnaître que les devins sarrasins sont efficaces : dans le Moniage Guillaume, Macabrant prédit les malheurs qui surviendront lorsque Guillaume sera retrouvé, ce que confirme la suite de la chanson ${ }^{35}$. Dans Gormont et Isembart, au cours de la bataille, lorsque l'issue funeste se fait sentir, Isembart se rappelle ce que lui avait prédit un devin sarrasin : le sort qu'on lui avait annoncé est désormais scellé ${ }^{36}$. Ainsi, la divination n'est pas discréditée, puisque ses prédictions se vérifient, mais les pratiques oraculaires ne suffisent pas néanmoins à infléchir le cours des événements et à sauver les Sarrasins de leur sort, puisqu'elles prévoient toujours la défaite ultime des païens. C'est là un moyen de montrer que les Chrétiens servent la juste cause, dans la mesure où ils sont soutenus par Dieu vers la victoire, alors que les Sarrasins sont condamnés à être vaincus, en dépit d'oracles efficaces qui, au lieu de les soutenir, scellent leur perte : l'acuité des devins sarrasins ne signifie pas en effet que le bon droit se trouve de leur côté.

En outre, l'habitude de grimer les devins sous des traits sarrasins n'est pas spécifique aux chansons de geste : une telle dichotomie opposant prophètes inspirés chrétiens et savants devins sarrasins se retrouve par exemple dans le Roman de Mahomet d'Alixandre dou Ponts, lequel se propose de relater la vie du prophète païen dont le récit est emprunté, paraît-il, à un musulman sincèrement converti au catholicisme. Mahomet est présenté comme un expert des arts libéraux et en particulier de

${ }^{35}$ «Tel cose i vi qui mie ne m'agree,/Dont cent mil Turc avront l'ame sevree, /S'en perderons toute nostre contree./Tout pour Guillaume ert no terre gastee, /Et recevrons pour lui tele soldee : /Tout en morrons au trençant de l'espee, /Jou en avrai l'ame del cors sevree », Le Moniage Guillaume II, éd. cit., v. 2864-2870.

${ }^{36}$ « e ! jal me dist un Sarrazin, /ultre la mer, qui en sorti, /si jeo veneie en cest pais, /que jeo serraie u mort u pris. /Or sai jeo bien que il veir dist », Gormont et Isembart, éd. Alphonse Bayot, Paris, Champion, 1914, v. 636-640. 
l'astrologie, cette précision n'étant guère étonnante dans la mesure où les Arabes passaient pour maîtres ès arts divinatoires et magiques. Or Mahomet, dans sa jeunesse, rencontre un ermite chrétien qui, en l'apercevant, est immédiatement saisi d'une vision : il annonce alors au jeune homme qu'il détruira les sacrements de la foi chrétienne, en particulier le mariage, le baptême, l'extrême-onction, etc. ${ }^{37}$. Cette scène est particulièrement intéressante en ce qu'elle inverse les rôles, le prophète étant non pas Mahomet mais l'ermite, qui bénéficie à ce moment précis du don de prescience. La suite du roman ne fait que développer l'imposture à laquelle se livre Mahomet, présenté comme un faux prophète, rompu à toutes les bassesses et à toutes les ruses. S'il ne met pas en pratique ses aptitudes d'astrologue dans le roman et ne fait pas ainsi figure de devin, c'est surtout parce qu'il s'efforce d'être considéré comme un prophète. Et lorsqu'il est surpris par une crise d'épilepsie en plein banquet, il transforme cette infirmité physique en signe d'élection divine en prétendant qu'un ange s'est posé sur lui, ce qui est une parodie grotesque des songes prémonitoires envoyés par Dieu aux Chrétiens ${ }^{38}$ dans les chansons de geste.

\section{De la prophétie aux prédictions autorisées}

Si la littérature se sert des Sarrasins pour mettre en scène la divination, notamment parce que cela permet de construire une opposition entre deux camps, deux peuples, deux religions, la question du rapport entre divination et religion se pose néanmoins pour les textes divinatoires

\footnotetext{
${ }^{37}$ Alixandre Dou Ponts, Le Roman de Mahomet, éd. Yvan G. Lepage, Paris, Klincsieck, 1977, v. 93-180.

${ }^{38}$ À ce titre, on peut souligner la similitude évidente entre le Roman de Mahomet et la Chanson de Roland qui nous pousse à dire qu'il s'agit bien là d'une parodie : l'ange invoqué par les deux textes, l'un pour justifier l'épilepsie, l'autre pour protéger Charlemagne et transmettre les visions divines, n'est autre que l'archange Gabriel. On se souviendra aussi que Jibrīl est justement celui qui transmet le Coran à Mahomet, impliquant donc une lecture doublement parodique de cette scène.
} 
qui circulent en Europe, dans la mesure où la divination, contrairement à ce que pourraient laisser entendre les œuvres littéraires, coexiste avec le christianisme dans l'Occident médiéval. Comme l'indique l'emploi de la prophétie dans la littérature médiévale en effet, la prédiction semble acceptable lorsqu'elle est autorisée par Dieu. Or, ce souci de justification est perceptible dans les manuscrits divinatoires.

Sans revenir sur le long débat à propos de la liberté et du destin qui a longtemps occupé les théologiens, ou sans se demander si les prédictions des devins sont soufflées par des démons ou par une intelligence malade, nous pouvons souligner que les textes divinatoires en français essaient autant que possible de gagner une légitimité chrétienne. Ils s'ouvrent et s'achèvent souvent par une prière rappelant le bien fondé religieux de la technique divinatoire employée ${ }^{39}$. Ces prières peuvent se retrouver à plusieurs reprises dans un même manuscrit, preuve s'il en est du désir de paraître conforme aux prescriptions de l'Église. En outre, le crédit de la technique oraculaire peut venir de sa figure tutélaire, ce qui est particulièrement vrai du traité oniromantique intitulé Les Songes Daniel le prophete, traduction de l'opuscule latin Somnia Danielis. L'extension du titre dans la traduction en langue vernaculaire marque bien une forme d'insistance sur la paternité religieuse et même biblique revendiquée de l'ouvrage ${ }^{40}$. Parfois, pour des techniques divinatoires uniquement fondées sur le hasard, il est plus difficile de trouver la moindre trace d'une quelconque légitimité chrétienne : l'ouvrage repose alors sur le prestige de

39 «En l'onor de Dieu Pere, Filz et Saint Esperit, qui forma ciel, terre et toutes autres choses et voult demoustrer a home par la science d'astrologie la verité des choses passees, presentes et a venir. [...] Benoit soit notre seignor Jhesu Crist, qui dona a home la connoissance de savoir les choses celestianes. Dieu, le Souverain Creator de toutes choses, ordena le soleil u mellieu des esperes avecques 3 planetes au dessus et 3 au dessous », Geomantia, Cambridge, Wren Library (Trinity College), ms. O.9.35, $\mathrm{f}^{\mathrm{o}} 1$.

${ }^{40}$ Pour quelques-unes des visions de Daniel qui expliquent pourquoi certains ouvrages oniromantiques se réclament de ce prophète, on pourra se reporter à la Bible, Daniel II, 19 ; VII, 1 ; VIII, 1 ; X, 7-11. 
son cadre référentiel, c'est-à-dire sur les personnages qu'il cite comme autant de cautions de ses prédictions. Ainsi, le Dodechedron, livre de sorts fondé par nature sur l'aléatoire, organise ses séries de réponses en douze catégories, chacune placée sous le patronage d'un roi illustre et d'une sibylle - prophétesse des temps païens fameuse au Moyen Âge puisqu'on lui attribue l'annonciation de la venue de Jésus-Christ ${ }^{41}$. De la sorte, le Dodechedron cesse de n'être qu'un livre de sorts, ses prédictions bénéficiant de la caution de personnages illustres pleinement autorisés par l'Église.

D'autres textes utilisent une méthode plus subreptice pour ne pas paraître suspects : ils se teintent d'une coloration astrologique, parce que l'astrologie est considérée comme une science, inscrite dans le cursus universitaire et familière de la cour des rois et des princes ${ }^{42}$. C'est ce prestige de l'astrologie, acceptée par toute la société, que revendique explicitement la géomancie du manuscrit O.9.35 de la Wren Library à Cambridge, par exemple, lequel affirme dès le prologue la parenté de cet art avec l'astrologie, autorisée par Dieu ${ }^{43}$. Ensuite, toute la première partie de l'ouvrage s'applique à décrire de nombreuses propriétés empruntées à

${ }^{41}$ Pour une étude approfondie de l'appropriation par l'Église de la figure païenne de la Sibylle, voir Julien Abed, La Parole de la sibylle. Fable et prophétie à la fin du Moyen Âge, thèse de doctorat, Université Paris-Sorbonne, 2010.

${ }^{42}$ Néanmoins, l'astrologie n'est pas reconnue d'utilité publique et reste essentiellement une affaire privée. Sur cette question en particulier, voir « Dans les cours princières : la faveur inégale de l'astrologie et des astrologues ", dans Jean-Patrice Boudet, Entre science et nigromance. Astrologie, divination et magie dans l'Occident médiéval (XII $-X V^{e}$ siècles), op. cit. ; et aussi «Magicians at Court» dans Richard Kieckhefer, Magic in the Middle Ages, op. cit., p. 97-100 ; ainsi que Doris Ruhe, « La divination au Moyen Âge. Théories et pratiques », dans Moultes obscures paroles : études sur la prophétie médiévale, dir. Richard Trachsler, Paris, Presses de l'Université ParisSorbonne, 2007, p. 25.

43 « et pour cen que c'est trop forte science et pour cen que d'icelle l'en ne puet ouvrer fors par instrumens tres soutils et gracieulz, [Dieu] voult que toutes ces choses fussent seues par l'art des poins qui est nommé geomancie, qui est estraite et vraie fille d'astrologie, par laquele l'en puet savoir les choses passees, presentes et a venir plus legierement et plus briefment », Geomantia, Cambridge, Wren Library (Trinity College), ms. O.9.35, $\mathrm{f}^{\mathrm{o}} 1$. 
l'astrologie (valeurs des différentes maisons, influences planétaires, aspects d'opposition, de trigone, de quadrature, aspects de mutation, conjonction, etc.) qui apparaissent comme autant de justifications de la géomancie ; sans cet apparat astrologique, on se rendrait bien compte que la technique ne se fonde en vérité que sur le tracé aléatoire de lignes de points, rien de plus. Dans ce cas, le recours à l'astrologie permet de donner du corps et de la légitimité à cet art divinatoire.

Néanmoins, il ne faudrait pas croire que ces tentatives d'autojustification aient été obligatoires et contraintes. Elles sont peut-être tout simplement le fait de devins chrétiens soucieux de concilier leur pratique et leur foi. Elles sont peut-être aussi le fait de devins pragmatiques qui comprenaient très bien l'intérêt de donner de la légitimité à leur pratique pour bénéficier d'un effet persuasif maximal sur leurs consultants. Dans tous les cas, elles ne sont pas signes de la peur d'une répréhension par l'Église. De nombreuses études ont déjà montré que les clercs pratiquaient plus ou moins extensivement la divination ${ }^{44}$ - certains utilisaient par exemple la sphère de Pythagore pour savoir s'il fallait administrer l'extrême onction à un malade, ou non ${ }^{45}$ - et ont montré aussi que les seigneurs commanditaires des manuscrits n'avaient que faire des positions théoriques de quelques théologiens. Cette tolérance envers la divination était d'ailleurs d'autant plus grande que les pratiques oraculaires étaient tout au plus accusées de vanité ; les choses en allaient tout autrement pour la nigromance.

En définitive, malgré le caractère protéiforme de «la» divination médiévale qui est largement renouvelée par la science des Arabes et qui

\footnotetext{
${ }^{44} \mathrm{Si}$ bien qu'il aurait même existé un «clerical underground» s'adonnant aux arts divinatoires. Voir Richard Kieckhefer, Magic in the Middle Ages, op. cit., p. 156.

${ }^{45}$ Charles Singer, From Magic to Science : Essays on the Scientific Twilight, London, Benn, 1928, p. 146.
} 
bénéficie d'un essor considérable à partir du $\mathrm{XIV}^{\mathrm{e}}$ siècle, la variété des arts divinatoires n'est que très partiellement reflétée dans la littérature de l'époque. Il semble y avoir un manque d'intérêt évident pour le détail des pratiques oraculaires, ce qui explique pourquoi les auteurs se contentent de dire que le devin «jette ses sorts». Les devins en effet sont plutôt appréciés pour leur rôle fonctionnel dans la narration, et puisque leurs prédictions se réalisent toujours, on ne s'embarrasse pas de détails concernant les arts divinatoires mis en œuvre. Peut-être est-ce également un moyen de créer l'étonnement, en laissant à d'autres la connaissance de ce qui paraît merveilleux pour le profane. Néanmoins, la littérature souligne aussi la frontière qui sépare les divinations autorisées par Dieu, lesquelles s'apparentent à des prophéties puisque ce sont des prédictions inspirées, et celles qui sont le produit d'un ensemble de techniques savantes, résultats d'esprits laborieux et industrieux. Or, un certain nombre de manuscrits de nature divinatoire s'efforce justement d'acquérir une légitimité religieuse, en s'appuyant en particulier sur le patronage de figures bibliques ou en se reposant en large partie sur le prestige de l'astrologie, qui n'attire pas les foudres de la jurisprudence cléricale. 